This item was submitted to Loughborough's Research Repository by the author.

Items in Figshare are protected by copyright, with all rights reserved, unless otherwise indicated.

\title{
Asymptotic estimation of state and faults for linear systems with unknown perturbations
}

PLEASE CITE THE PUBLISHED VERSION

https://doi.org/10.1016/j.automatica.2020.108955

PUBLISHER

Elsevier BV

VERSION

AM (Accepted Manuscript)

PUBLISHER STATEMENT

This paper was accepted for publication in the journal Automatica and the definitive published version is available at https://doi.org/10.1016/j.automatica.2020.108955

LICENCE

CC BY-NC-ND 4.0

\section{REPOSITORY RECORD}

Lan, Jianglin. 2020. "Asymptotic Estimation of State and Faults for Linear Systems with Unknown Perturbations”. Loughborough University. https://hdl.handle.net/2134/12083184.v1. 


\title{
Asymptotic estimation of state and faults for linear systems with unknown perturbations
}

\author{
Jianglin Lan \\ Department of Aeronautical and Automotive Engineering, Loughborough University, Leicestershire, LE11 3TU, UK.
}

\begin{abstract}
It is challenging to achieve asymptotic estimation of state and actuator faults for systems with unknown perturbations in both the state dynamics and output measurements. To address this, an adaptive sliding mode unknown input observer (ASMUIO) is developed under a mild rank condition of the perturbation distribution matrices. The key idea is to estimate the perturbations simultaneously with the state and faults, which then vanishes the perturbation effects from the estimation error dynamics. Existence conditions and rigorous feasibility proof of the proposed ASMUIO are given. A simulation example is provided to demonstrate the design efficacy in comparison with existing approaches.
\end{abstract}

Key words: Asymptotic estimation; fault estimation; perturbation; descriptor system; unknown input observer.

\section{Introduction}

Fault estimation (FE) can provide direct reconstruction of fault signals and thus plays an important role in the active fault-tolerant control (FTC) framework. FE designs are effective unless they have good robustness against perturbations including external disturbance and/or system uncertainty, acting on the state dynamics and/or output measurements.

There are mainly three types of approaches in the literature to enhance FE robustness. The first type is attenuation approach, which suppresses the perturbations using $H_{\infty}$ optimization. It is conservative but widely used in the literature, see for example the extended state observer (Gao \& Ding, 2007) and augmented state unknown input observer (ASUIO) (Lan \& Patton, 2016). The second type is decoupling approach, which removes from the estimation error dynamics the perturbations whose distribution matrix satisfy the matching rank condition (Gao et al., 2016). The sliding mode observers (SMOs) for FE (Huang et al., 2016; Yan \& Edwards, 2007) also use the spirit of decoupling to realize insensitivity to the state perturbations that satisfy a similar matching condition. The decoupling approach has limited applicability because usually only partial perturbations can meet the matching condition. The non-

\footnotetext{
* The material in this paper was not presented at any conference. Corresponding author Jianglin Lan.

Email address: j.lan@lboro.ac.uk (Jianglin Lan).
}

decoupling part is attenuated using $H_{\infty}$ optimization (Gao et al., 2016; Huang et al., 2016; Yan \& Edwards, 2007). The third type is reconstruction approach, which eliminates the perturbation effects by estimating them. An adaptive sliding mode unknown input observer (ASMUIO) is proposed in Lan \& Patton (2018) to achieve asymptotic estimation of state, actuator faults and perturbations for linear systems. Different from other SMO based FE designs, the ASMUIO can handle perturbations in both state and outputs. Moreover, the estimated perturbations can be used for robust system control.

This paper proposes a new ASMUIO to achieve asymptotic estimation of state, actuator faults and perturbations, where the perturbation distribution matrices satisfy a less conservative rank condition compared with Lan \& Patton (2018). Moreover, the proposed ASMUIO contains the one in Lan \& Patton (2018) as a special case. Designing the ASMUIO under such a relaxed rank condition relies on reformulating the original system as an infinitely observable descriptor system (Dai, 1989), where partial perturbations are regarded as auxiliary state and the rest as virtual faults. The observer design is then described based on Lan \& Patton (2018) with existence conditions and feasibility proof provided.

The rest of this paper is organized as follows. Section 2 states the problem. Section 3 presents the system reformulation. Section 4 describes the observer design. Section 5 provides a simulation example. Section 6 draws the conclusions. 


\section{Problem statement}

Consider a class of linear systems represented by

$\dot{x}=A x+B u+F f+D_{1} d$

$y=C x+D_{2} d$

where $x \in \mathbb{R}^{n}, u \in \mathbb{R}^{m}$ and $y \in \mathbb{R}^{p}$ are the state, control input and output, respectively. $f \in \mathbb{R}^{l}$ is the energy bounded actuator fault representing additive, component or partial loss of effectiveness faults (Lan \& Patton, 2016). $d \in \mathbb{R}^{q}$ is the perturbation representing a large class of signals, e.g. disturbance, uncertainty, additive nonlinearity and sensor bias, for which more details can be found in Chapter 5 of Chen \& Patton (1999). A, B, $F, D_{1}, C$ and $D_{2}$ are known constant matrices of appropriate dimensions. The system satisfies $\operatorname{rank}(F)=l$, $\operatorname{rank}(C)=p$, and the following assumptions:

Assumption 2.1 $\operatorname{rank}\left[\begin{array}{ccc}s I-A & F & D_{1} \\ C & 0 & D_{2}\end{array}\right]=n+q+l$, $\forall s \in \mathbb{C}, \operatorname{Re}(s) \geq 0$.

Assumption $2.2 \operatorname{rank}\left[\begin{array}{ll}D_{1}^{\top} D_{2}^{\top}\end{array}\right]^{\top}=q$.

Assumption $2.3 \operatorname{rank}\left[\begin{array}{ccc}C F & C D_{1} & D_{2} \\ 0 & D_{2} & 0\end{array}\right]=\operatorname{rank}\left(D_{2}\right)+$ $\operatorname{rank}\left[\begin{array}{cc}F & D_{1} \\ 0 & D_{2}\end{array}\right]$

This paper aims to design an observer for the system (1) to obtain asymptotic estimation of $x$ and $f$, and realize reconstruction of $d$ as a way of completely eliminating the perturbation effects.

Assumptions 2.1 and 2.3 coincide respectively with the minimum phase condition and matching condition that ensure strong* detectability of the system (Hautus, 1983). These conditions are well-known sufficient conditions for achieving asymptotic state estimation in the presence of disturbances (which include faults and perturbations here). Assumption 2.2 is further needed for the proposed observer design to achieve simultaneously asymptotic estimation of state, faults and perturbations, which has not been discussed in the literature. Moreover, Assumption 2.2 is more general than the one $\left(\operatorname{rank}\left(D_{2}\right)=q\right)$ in Lan \& Patton (2018), and contains the latter as a special case. The condition $\operatorname{rank}\left(D_{2}\right)=q$ is satisfied only when all the perturbations appear at the output measurements. However, under the new rank condition, the perturbations acting on the state and output can be different. For example, the perturbations can include a state disturbance and a sensor bias. Therefore, the observer designed based on this new rank condition is applicable for a wider class of systems.
Without loss of generality, this work focuses on the case when $\operatorname{rank}\left(D_{2}\right)=r<q$. In such a case, following the same system augmentation strategy described in Lan \& Patton (2018) gives an infinitely unobservable descriptor system, for which no asymptotic observer exists. To overcome this problem, a system reformulation is performed in Section 3 to obtain an infinitely observable descriptor system that admits an asymptotic observer.

\section{System reformulation}

Since $\operatorname{rank}\left(D_{2}\right)=r<q$, the singular value decomposition of $D_{2}$ is given by $D_{2}=U_{d} \Sigma_{d} V_{d}^{\top}$, where $U_{d} \in \mathbb{R}^{p \times p}$ and $V_{d} \in \mathbb{R}^{q \times q}$ are orthogonal matrices, and $\Sigma_{d}=$ $\operatorname{diag}\left\{\Sigma_{r}, 0_{(p-r) \times(q-r)}\right\}$ is a block diagonal matrix with the diagonal matrix $\Sigma_{r} \in \mathbb{R}^{r \times r}$ containing all the $r$ singular values of $D_{2}$ on the diagonal. Define the terms

$V_{d}^{\top} d=\left[\begin{array}{l}d_{1} \\ d_{2}\end{array}\right] \begin{aligned} & \uparrow q \\ & \uparrow q-r\end{aligned}, U_{d}^{\top} y=\bar{y}$,
$D_{1} V_{d}=\left[\begin{array}{ll}D_{11} & D_{12}\end{array}\right], U_{d}^{\top} C=\tilde{C}$.

By using (2), the system (1) is rewritten as

$\dot{x}=A x+B u+\mathcal{F} \bar{f}+D_{11} d_{1}$

$\bar{y}=\tilde{C} x+\bar{D}_{2} d_{1}$

where $\bar{f}=\left[\begin{array}{ll}f^{\top} & d_{2}^{\top}\end{array}\right]^{\top}, \mathcal{F}=\left[\begin{array}{ll}F & D_{12}\end{array}\right]$ and $\bar{D}_{2}=\left[\begin{array}{ll}\Sigma_{r}^{\top} & 0\end{array}\right]^{\top}$.

The system (3) is rearranged into the descriptor form

$\mathcal{E} \dot{\chi}=\mathcal{A} \chi+\mathcal{B} u+\mathcal{F} \bar{f}$

$\bar{y}=\mathcal{C} \chi$

where $\chi=\left[\begin{array}{ll}x^{\top} & d_{1}^{\top}\end{array}\right]^{\top}, \mathcal{E}=\left[\begin{array}{ll}I_{n} & 0_{n \times r}\end{array}\right], \mathcal{A}=\left[\begin{array}{ll}A & D_{11}\end{array}\right]$, $\mathcal{B}=B$ and $\mathcal{C}=\left[\begin{array}{ll}\tilde{C} & \bar{D}_{2}\end{array}\right]$. Here $d_{1}$ is treated as auxiliary state and $d_{2}$ as virtual faults to be estimated together with the real fault $f$. If following Lan \& Patton (2018) by regarding the entire vector $d$ as auxiliary state, then $\mathcal{E}=\left[\begin{array}{ll}I_{n} & 0_{n \times q}\end{array}\right], \mathcal{A}=\left[\begin{array}{ll}A & D_{1}\end{array}\right], \mathcal{F}=F$ and $\mathcal{C}=\left[\begin{array}{ll}C & D_{2}\end{array}\right]$, which will result in an infinitely unobservable descriptor system (i.e. the violation of Corollary 3.1).

Corollary 3.1 Under Assumptions 2.1 - 2.3, the descriptor system (4) holds the following properties:

$$
\begin{aligned}
\operatorname{rank}\left[\begin{array}{l}
\mathcal{E} \\
\mathcal{C}
\end{array}\right] & =n+r, \\
\operatorname{rank}\left[\begin{array}{cc}
\mathcal{E} & \mathcal{F} \\
\mathcal{C} & 0
\end{array}\right] & =n+l+q,
\end{aligned}
$$

$\operatorname{rank}\left[\begin{array}{cr}s \mathcal{E}-\mathcal{A} & \mathcal{F} \\ \mathcal{C} & 0\end{array}\right]=n+l+q, \forall s \in \mathbb{C}, \operatorname{Re}(s) \geq 0$. 
Proof 3.1 Since $\operatorname{rank}\left(\bar{D}_{2}\right)=r$, it holds that

$$
\operatorname{rank}\left[\begin{array}{l}
\mathcal{E} \\
\mathcal{C}
\end{array}\right]=\operatorname{rank}\left[\begin{array}{cc}
I_{n} & 0 \\
\tilde{C} & \bar{D}_{2}
\end{array}\right]=n+r .
$$

In view of the structure of $\bar{D}_{2}$ in (3), it follows that

$$
\operatorname{rank}\left[\begin{array}{cc}
D_{11} & D_{12} \\
\bar{D}_{2} & 0
\end{array}\right]=r+\operatorname{rank}\left(D_{12}\right)
$$

According to Assumption 2.2 and (2), one gets

$$
\operatorname{rank}\left[\begin{array}{cc}
D_{11} & D_{12} \\
\bar{D}_{2} & 0
\end{array}\right]=\operatorname{rank}\left\{\left[\begin{array}{cc}
I_{n} & 0 \\
0 & U_{d}^{\top}
\end{array}\right]\left[\begin{array}{c}
D_{1} \\
D_{2}
\end{array}\right] V_{d}\right\}=q .
$$

Combining the above equation with (8) gives $\operatorname{rank}\left(D_{12}\right)=$ $q-r$ and $\operatorname{col}\left(\tilde{C} D_{11}\right) \subseteq \operatorname{col}\left(\bar{D}_{2}\right)$, where $\operatorname{col}(\cdot)$ represents the column space spanned by the matrix. This implies that

$$
\operatorname{rank}\left[\bar{D}_{2} \tilde{C} F \tilde{C} D_{1} V_{d}\right]=\operatorname{rank}\left[\bar{D}_{2} \tilde{C} F \tilde{C} D_{12}\right] .
$$

Since $U_{d}$ and $V_{d}$ are non-singular, it holds that

$$
\operatorname{rank}\left[\bar{D}_{2} \tilde{C} F \tilde{C} D_{1} V_{d}\right]=\operatorname{rank}\left[D_{2} C F C D_{1}\right]
$$

By using Assumptions 2.1 - 2.3, one has

$$
\operatorname{rank}\left[C F C D_{1} D_{2}\right]=\operatorname{rank}\left[\begin{array}{cc}
F & D_{1} \\
0 & D_{2}
\end{array}\right]=l+q .
$$

Combining (9) and (10) yields $\operatorname{rank}\left[\bar{D}_{2} \tilde{C} F \tilde{C} D_{12}\right]=$ $l+q$. This results in

$$
\begin{aligned}
\operatorname{rank}\left[\begin{array}{cc}
\mathcal{E} & \mathcal{F} \\
\mathcal{C} & 0
\end{array}\right] & =\operatorname{rank}\left\{\left[\begin{array}{cc}
I_{n} & 0 \\
-\tilde{C} & I_{p}
\end{array}\right]\left[\begin{array}{cccc}
I_{n} & 0 & F & D_{12} \\
\tilde{C} & \bar{D}_{2} & 0 & 0
\end{array}\right]\right\} \\
& =\operatorname{rank}\left[\begin{array}{cccc}
I_{n} & 0 & F & D_{12} \\
0 & \bar{D}_{2} & \tilde{C} F & \tilde{C} D_{12}
\end{array}\right] \\
& =n+l+q .
\end{aligned}
$$

Under Assumption 2.1, it can be derived that

$$
\begin{aligned}
\operatorname{rank}\left[\begin{array}{cr}
s \mathcal{E}-\mathcal{A} & \mathcal{F} \\
\mathcal{C} & 0
\end{array}\right] & =\operatorname{rank}\left\{X_{1}\left[\begin{array}{ccc}
s I_{n}-A & F & D_{1} \\
C & 0 & D_{2}
\end{array}\right] X_{2}\right\} \\
& =n+l+q
\end{aligned}
$$

where $X_{1}=\operatorname{diag}\left\{I_{n}, U_{d}^{\top}\right\}$ and $X_{2}=\operatorname{diag}\left\{I_{n}, I_{l}, V_{d}\right\}$ are full rank. Therefore, (7) is satisfied.

The properties (5) and (7) ensure that the system (4) is infinitely observable (Dai, 1989), and (6) further ensures that $\bar{f}$ can be fully reconstructed. Therefore, there exists an observer for (4) to estimate accurately $\chi$ and $\bar{f}$, and subsequently $x, f$ and $d$ of the original system (1).
Remark 3.1 FE design for descriptor systems is studied in Chan et al. (2017) following a similar reformulation strategy. However, there are a few differences between this paper and their work. First, both state and output perturbations are considered in this paper, while only state perturbation is studied in their work. Second, the matrix $\mathcal{E}$ in (4) is rectangular, while it is square in their work. Third, the canonical form SMO (Yan 8 Edwards, 2007) is used in their work to estimate the state and faults, while the Walcott-Żak form SMO (Huang et al., 2016) is adopted here.

\section{Observer design}

Since the descriptor system (4) and the one in Lan \& Patton (2018) share the same form, the observer designs for them are similar. Therefore, this section only briefly describes the observer design, while its focus is to provide a rigorous feasibility proof of asymptotic estimation, which is missing in Lan \& Patton (2018).

The ASMUIO is designed as

$$
\begin{aligned}
& \dot{z}=N z+J u+L \bar{y}+W v \\
& \hat{\chi}=z+H \bar{y}
\end{aligned}
$$

where $z \in \mathbb{R}^{n+r}$ is the observer state and $\hat{\chi} \in \mathbb{R}^{n+r}$ is the estimate of $\chi . N, J, L, W$ and $H$ are design matrices. The sliding switching function $v$ is designed as $v=\hat{\rho} \operatorname{sign}\left(e_{y}\right)$, with $e_{y}=\bar{y}-\mathcal{C} \hat{\chi}$ and a scalar gain $\hat{\rho}$ governed by

$$
\dot{\hat{\rho}}=\sigma_{0}\left\|e_{y}\right\|, \hat{\rho}(0)=0,
$$

with a given positive constant $\sigma_{0}$. A larger $\sigma_{0}$ can increase the estimation accuracy of $\bar{f}$ but leading to a high gain $\hat{\rho}$ and possible bigger overshoots at transients. A trade-off can be realized via trial and error. The existence conditions of asymptotic estimation are given below.

Theorem 4.1 Under Assumptions 2.1 - 2.3, the observer (11) can achieve asymptotic estimation of $x, f$ and $d$, if there is a symmetric positive definite matrix $P$, matrices $T$ and $Q$, and a positive scalar $\alpha$ such that

$$
\begin{aligned}
& P N+N^{\top} P+2 \alpha P<0, \\
& P T \mathcal{F}=\mathcal{C}^{\top} Q .
\end{aligned}
$$

Then the estimates of $x, f$ and $d$ are given as:

$\hat{x}=\left[\begin{array}{ll}I_{n} & 0\end{array}\right] \hat{\chi}, \hat{f}=\left[\begin{array}{ll}I_{l} & 0\end{array}\right] \hat{\bar{f}}, \hat{d}=V_{d}\left[\begin{array}{l}\hat{d}_{1} \\ \hat{d}_{2}\end{array}\right], \hat{d}_{1}=\left[\begin{array}{ll}0 & I_{r}\end{array}\right] \hat{\bar{x}}$,

$\hat{d}_{2}=\left[\begin{array}{ll}0 & I_{q-r}\end{array}\right] \hat{\bar{f}}, \hat{\bar{f}}=\left(Q^{\top} \mathcal{C} P^{-1} \mathcal{C}^{\top} Q\right)^{-1} Q^{\top} \mathcal{C} P^{-1} \mathcal{C}^{\top} v_{\text {eq }}$, where $v_{e q}$ is the low pass filtering of $v$.

Proof 4.1 The proof is based on Lyapunov stability theory and similar to that of Theorem 3.1 in Lan \& Patton (2018). Hence, it is only briefly discussed below. By 
defining $e=\chi-\hat{\chi}$ and the matrix equations

$$
\begin{aligned}
T \mathcal{A}-N T \mathcal{E}-L \mathcal{C} & =0, \\
T \mathcal{B}-J & =0, \\
I_{n+r}-H \mathcal{C}-T \mathcal{E} & =0,
\end{aligned}
$$

then the estimation error system is derived as

$$
\dot{e}=N e+T \mathcal{F} \bar{f}-W v .
$$

The condition (13) is then given to make $N$ stable. Different from Lan $\&$ Patton (2018), the term $2 \alpha P$ is introduced to improve the decay rate of $e(t)$. Since $v$ can only compensate a matched term, the matrix $W$ is designed as $W=P^{-1} \mathcal{C}^{\top}$ and a matrix $Q$ is introduced to make $P T \mathcal{F}=\mathcal{C}^{\top} Q$ and thus $T \mathcal{F} \bar{f}=P^{-1} \mathcal{C}^{\top} Q \bar{f}$. This ensures that $T \mathcal{F} \bar{f}-W v=P^{-1} \mathcal{C}^{\top}(Q \bar{f}-v)$. Therefore, $v$ can be designed to compensate the matched term $Q \bar{f}$ under satisfaction of the equality condition (14).

Lemma 4.1 The conditions (13) and (14) in Theorem 4.1 are always feasible under Assumptions 2.1 - 2.3.

Proof 4.2 Multiplying (4) with $T$ and using (17) gives

$$
\begin{aligned}
\dot{\chi} & =T \mathcal{A} \chi+T \mathcal{B} u+T \mathcal{F} \bar{f}+H \dot{\bar{y}} \\
\bar{y} & =\mathcal{C} \chi .
\end{aligned}
$$

By Lemma 3 of Corless \& Tu (1998), there is a symmetric positive definite matrix $\check{P}$ and a matrix $\check{L}$ such that

$$
\begin{gathered}
\check{P}(T \mathcal{A}-\check{L} \mathcal{C})+(T \mathcal{A}-\check{L} \mathcal{C})^{\top} \check{P}<0, \\
\check{P} T \mathcal{F}=\mathcal{C}^{\top} Q,
\end{gathered}
$$

if and only if the following conditions hold:

C1: $\operatorname{rank}(\mathcal{C} T \mathcal{F})=\operatorname{rank}(T \mathcal{F})=l+q-r$.

C2: All the invariant zeros of $(T \mathcal{A}, T \mathcal{F}, \mathcal{C})$ are stable.

The next step is to prove satisfaction of C1 and C2 under Assumptions 2.1 - 2.3. According to (17), it holds that

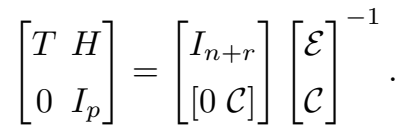

Hence, the left-hand side of (20) has full column rank $n+r$ and it follows from (6) that

$$
\begin{aligned}
\operatorname{rank}\left[\begin{array}{cc}
\mathcal{E} & \mathcal{F} \\
\mathcal{C} & 0
\end{array}\right] & =\operatorname{rank}\left\{\left[\begin{array}{cc}
T & H \\
0 & I_{p}
\end{array}\right]\left[\begin{array}{ll}
\mathcal{E} & \mathcal{F} \\
\mathcal{C} & 0
\end{array}\right]\right\} \\
& =\operatorname{rank}\left[\begin{array}{cc}
I_{n+r} & T \mathcal{F} \\
\mathcal{C} & 0
\end{array}\right] \\
& =n+l+q
\end{aligned}
$$

According to Lemma 1 in Yu \& Liu (2009), (21) is equivalent to the condition C1, which means that C1 holds.
By using (7), (17) and (20), it gives $\forall s \in \mathbb{C}, \operatorname{Re}(s) \geq 0$,

$$
\begin{aligned}
\operatorname{rank}\left[\begin{array}{rr}
s \mathcal{E}-\mathcal{A} & \mathcal{F} \\
\mathcal{C} & 0
\end{array}\right] & =\operatorname{rank}\left\{\left[\begin{array}{cc}
T & s H \\
0 & I_{p}
\end{array}\right]\left[\begin{array}{cr}
s \mathcal{E}-\mathcal{A} \mathcal{F} \\
\mathcal{C} & 0
\end{array}\right]\right\} \\
& =\operatorname{rank}\left[\begin{array}{cc}
s I_{n+r}-T \mathcal{A} T \mathcal{F} \\
\mathcal{C} & 0
\end{array}\right] \\
& =n+l+q .
\end{aligned}
$$

This means that the condition C2 holds. The above analysis shows that C1 and C2 are satisfied under Corollary 3.1. Hence, (18) and (19) are feasible under Assumptions 2.1 - 2.3. By using (15), (17) and $\bar{L}=L-N H$, one has $N=T \mathcal{A}-\bar{L} \mathcal{C}$. Defining $P=\check{P}$ and $\bar{L}=\check{L}$, then feasibility of (18) and (19) induces that of (13) and (14).

The conditions (13) and (14) are nonlinear of $P, N$ and $T$ and cannot be solved directly. As in Liu et al. (2017), a special solution of $T$ can be obtained from (17) and used to make (14) linear. However, this imposes design conservativeness. To solve (13) and (14) less conservatively, this paper adopts the parametrization approach from Lan \& Patton (2018). Based on (15) and (17), the matrices $T, H, N$ and $\bar{L}(\bar{L}=L-N H)$ are parametrized by two design matrices $Y_{1}$ and $Y_{2}$ as follows:

$$
\begin{aligned}
& T=T_{1}-Y_{1} T_{2}, H=H_{1}-Y_{1} H_{2}, \\
& N=N_{1}-Y_{2} N_{2}, \bar{L}=\bar{L}_{1}-Y_{2} \bar{L}_{2},
\end{aligned}
$$

where $T_{1}=\Omega_{1}^{\dagger} \Gamma_{1}, T_{2}=\left(I_{n+p}-\Omega_{1} \Omega_{1}^{\dagger}\right) \Gamma_{1}, H_{1}=$ $\Omega_{1}^{\dagger} \Gamma_{2}, H_{2}=\left(I_{n+p}-\Omega_{1} \Omega_{1}^{\dagger}\right) \Gamma_{2}, N_{1}=T \mathcal{A} \Omega_{2}^{\dagger} \Gamma_{3}, N_{2}=$ $\left(I_{n+r+p}-\Omega_{2} \Omega_{2}^{\dagger}\right) \Gamma_{3}, \bar{L}_{1}=T \mathcal{A} \Omega_{2}^{\dagger} \Gamma_{4}, \bar{L}_{2}=\left(I_{n+r+p}-\right.$ $\left.\Omega_{2} \Omega_{2}^{\dagger}\right) \Gamma_{4}, \Omega_{1}=\left[\begin{array}{l}\mathcal{E} \\ \mathcal{C}\end{array}\right], \Omega_{2}=\left[\begin{array}{c}I_{n+r} \\ \mathcal{C}\end{array}\right], \Gamma_{1}=\left[\begin{array}{c}I_{n} \\ 0\end{array}\right]$, $\Gamma_{2}=\left[\begin{array}{c}0_{n \times p} \\ I_{p}\end{array}\right], \Gamma_{3}=\left[\begin{array}{c}I_{n+r} \\ 0\end{array}\right], \Gamma_{4}=\left[\begin{array}{c}0_{(n+r) \times p} \\ I_{p}\end{array}\right]$.

Applying (22) to (13) and (14), and defining $Y=\left[Y_{1} Y_{2}\right]$ and $M=P Y$, it then gives the linear conditions (23) and (24) in Lemma 4.2. Once $P, Y_{1}$ and $Y_{2}$ are obtained from Lemma 4.2, all the observer gains $(N, J, L, W, H)$ can be determined using $(16),(22)$ and $W=P^{-1} \mathcal{C}^{\dagger}$.

Lemma 4.2 Under Assumptions 2.1 - 2.3, the observer (11) can achieve asymptotic estimation of $x, f$ and $d$, if there exists a symmetric positive definite matrix $P$, matrices $M$ and $Q$, and a positive scalar $\alpha$ such that

$$
\begin{gathered}
\left(P T_{1} \Phi-M T_{2 N}\right)+\left(P T_{1} \Phi-M T_{2 N}\right)^{\top}+2 \alpha P<0 \\
\left(P T_{1}-M \hat{T}_{2}\right) \mathcal{F}=\mathcal{C}^{\top} Q \\
\text { where } \Phi=\mathcal{A} \Omega_{2}^{\dagger}\left[\begin{array}{c}
I_{n+r} \\
0
\end{array}\right], T_{2 N}=\left[\begin{array}{c}
T_{2} \Phi \\
N_{2}
\end{array}\right], \hat{T}_{2}=\left[\begin{array}{c}
T_{2} \\
0
\end{array}\right] .
\end{gathered}
$$

Then the matrices $Y_{1}$ and $Y_{2}$ are obtained as 


$$
Y_{1}=P^{-1} M\left[\begin{array}{c}
I_{n+p} \\
0
\end{array}\right], Y_{2}=P^{-1} M\left[\begin{array}{c}
0 \\
I_{n+r+p}
\end{array}\right]
$$

Remark 4.1 The proposed observer can obtain asymptotic estimation of state, faults and perturbations, which is impossible for the observers using the attenuating approach (Lan \& Patton, 2016) or decoupling approach (Gao et al., 2016). The existence conditions of their observers and the proposed observer are different and summarized below. (i) The observer in Lan 8 Patton (2016) requires that all the invariant zeros of $(A, F, C)$ are stable, $\operatorname{rank}(F)=l$, and $l \leq p$. (ii) The observer in Gao et al. (2016) partitions $D_{1}$ as $D_{1}=\left[\check{D}_{11} \check{D}_{12}\right]$ corresponding to the partition $d=\left[\begin{array}{ll}\check{d}_{1}^{\top} & \check{d}_{2}^{\top}\end{array}\right]^{\top}$ with $\check{d}_{1} \in \mathbb{R}^{q_{1}}, \check{d}_{2} \in \mathbb{R}^{q_{2}}$ and $q_{1}+q_{2}=q$. It requires that all the invariant zeros of $\left(A,\left[\begin{array}{ll}F & \check{D}_{11}\end{array}\right], C\right)$ are stable, $\operatorname{rank}\left(C \check{D}_{11}\right)=\operatorname{rank}\left(\check{D}_{11}\right)=$ $q_{1}, \operatorname{rank}(F)=l$, and $l+q_{1} \leq p$. (iii) The proposed observer requires Assumptions 2.1 - 2.3 and $l+q \leq p$.

\section{Simulation example}

Adopting from Eugene et al. (2013) the normalized aircraft system in the form of (1) with the matrices

$A=\left[\begin{array}{ccc}-0.025 & 1.04 & -9.94 \\ 57.47 & 0 & 0 \\ 1.62 & 0 & 0\end{array}\right], B=\left[\begin{array}{cc}0.122 & -0.276 \\ -5.361 & 3.325 \\ 19.55 & -52.94\end{array}\right]$,
$F=\left[\begin{array}{c}0.122 \\ -5.361 \\ 19.55\end{array}\right], D_{1}=\left[\begin{array}{cc}0 & -0.276 \\ 0 & 3.325 \\ 0 & -52.94\end{array}\right], D_{2}=\left[\begin{array}{cc}0.1 & 0 \\ 0.2 & 0 \\ 1 & 0\end{array}\right]$,

and the state $x=\left[\begin{array}{ll}\beta & p r\end{array}\right]^{\top}$, control input $u=\left[\begin{array}{ll}\delta_{a} & \delta_{r}\end{array}\right]^{\top}$, output $y=x$ and perturbation $d=\left[\begin{array}{ll}d_{a} & d_{s}\end{array}\right]^{\top} . \beta$ is the slideslip angle, $p$ is the roll rate, $r$ is the yaw rate, $\delta_{a}$ is the aileron command and $\delta_{r}$ is the rudder command. The controller is given as

$$
u=\left[\begin{array}{ccc}
-6.841 & -0.2919 & 0.1227 \\
7.9536 & 0.1866 & -0.3261
\end{array}\right] x .
$$

The fault $f$ and the attacks $d_{a}$ and $d_{s}$ are characterized by $f(t)=0.5+\sin (2.5 \pi t), d_{a}(t)=0.5 \cos (2.5 t)+$ $\cos (2 t) \sin (\beta(t)) \cos (p(t))$, and $d_{s}(t)=\cos (\pi t)$.

This simulation system satisfies Assumptions 2.1 - 2.3. Comparative simulations are run for the three observers specified below. (i) The observer in Lan \& Patton (2016) using the attenuation approach, where $f$ is extended as auxiliary state while $d_{a}$ and $d_{s}$ are suppressed with the $H_{\infty}$ performance index $\gamma=9$. (ii) The observer in Gao et al. (2016) using the decoupling approach, where $f$ and $\dot{f}$ are extended as auxiliary state and $d_{a}$ is decoupled, while $d_{s}$ is attenuated with the $H_{\infty}$ performance index $\gamma=0.1$. (iii) The proposed observer with $\sigma_{0}=2500$ and the following gains solved from Lemma 4.2 with $\alpha=1$ :

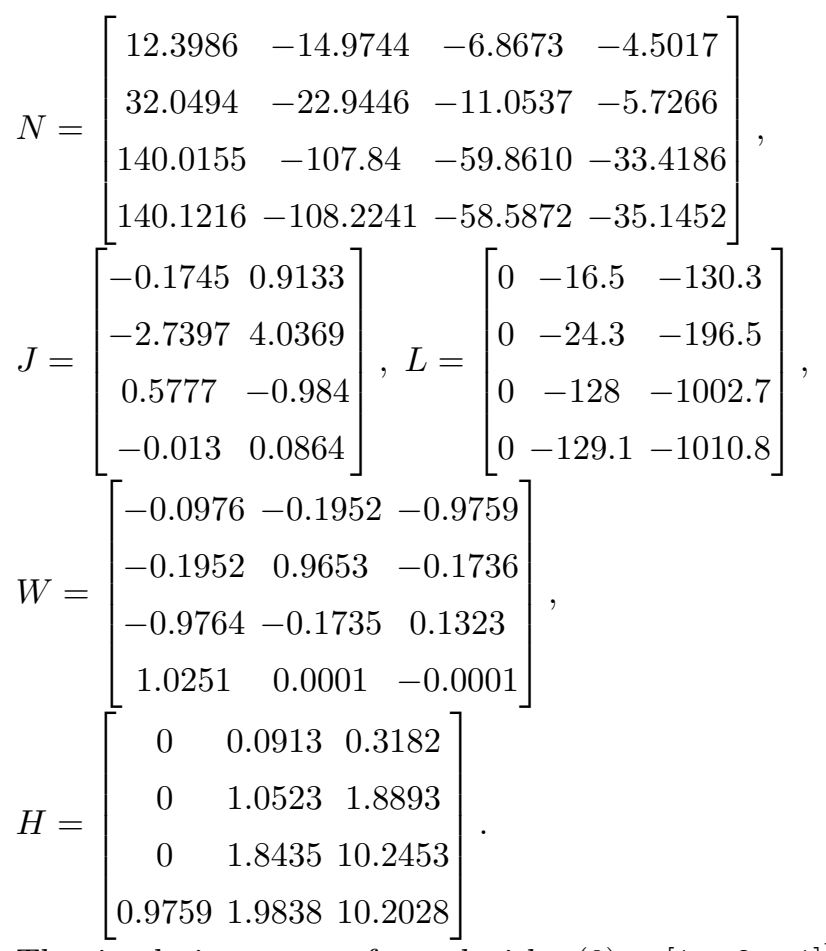

The simulations are performed with $x(0)=[1-2-1]^{\top}$ and zero initial observer state. The results in Fig. 1 show that the proposed observer achieves the best state and fault estimation, and accurate perturbation estimation.
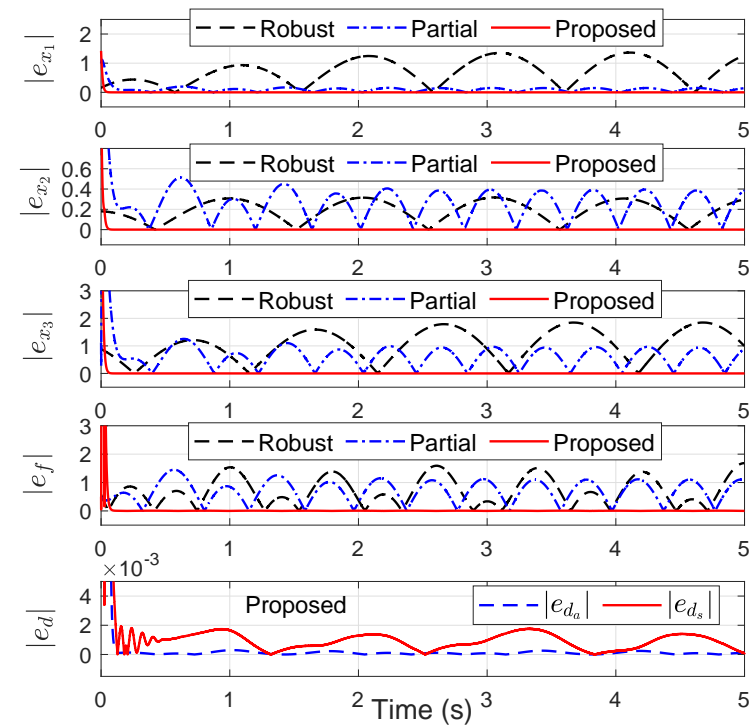

Fig. 1. Estimation errors of the three observers.

\section{Conclusion}

An adaptive sliding mode unknown input observer is proposed to obtain asymptotic estimation of state, faults and perturbations for linear systems with perturbations in both the state dynamics and outputs. Existence conditions of asymptotic estimation are provided based on a 
mild rank condition of the perturbation distribution matrices. Efficacy of the observer is verified through comparative simulations. Future work will consider observer designs for linear systems with invariant zeros on the imaginary axis, and for large-scale complex systems.

\section{References}

Chan, J. C. L., Tan, C. P., \& Trinh, H. (2017). Robust fault reconstruction for a class of infinitely unobservable descriptor systems. International Journal of Systems Science, 48, 1646-1655.

Chen, J., \& Patton, R. J. (1999). Robust model-based fault diagnosis for dynamic systems. Kluwer Academic Publishers.

Corless, M., \& Tu, J. (1998). State and input estimation for a class of uncertain systems. Automatica, 34, 757764 .

Dai, L. (1989). Singular control systems. Springer.

Eugene, L., Kevin, W., \& Howe, D. (2013). Robust and adaptive control with aerospace applications. Springer.

Gao, Z., \& Ding, S. X. (2007). Actuator fault robust estimation and fault-tolerant control for a class of nonlinear descriptor systems. Automatica, 43, 912-920.

Gao, Z., Liu, X., \& Chen, M. Z. (2016). Unknown input observer-based robust fault estimation for systems corrupted by partially decoupled disturbances. IEEE Transactions on Industrial Electronics, 63, 25372547.

Hautus, M. L. (1983). Strong detectability and observers. Linear Algebra and its Applications, 50, 353368.

Huang, Z., Patton, R. J., \& Lan, J. (2016). Sliding mode state and fault estimation for decentralized systems. In Variable-Structure Approaches (pp. 243281). Springer.

Lan, J., \& Patton, R. J. (2016). A new strategy for integration of fault estimation within fault-tolerant control. Automatica, 69, 48-59.

Lan, J., \& Patton, R. J. (2018). A decoupling approach to integrated fault-tolerant control for linear systems with unmatched non-differentiable faults. Automatica, 89, 290-299.

Liu, M., Zhang, L., \& Zheng, W. X. (2017). Fault reconstruction for stochastic hybrid systems with adaptive discontinuous observer and non-homogeneous differentiator. Automatica, 85, 339-348.

Yan, X.-G., \& Edwards, C. (2007). Nonlinear robust fault reconstruction and estimation using a sliding mode observer. Automatica, 43, 1605-1614.

Yu, J., \& Liu, Z. (2009). Fault reconstruction based on sliding mode observer for linear descriptor systems. In Proceedings of the Asian Control Conference (pp. 1132-1137). IEEE. 